\title{
A Novel Extension for On-demand Routing Protocol in Event-Driven Sensor Networks ${ }^{\star}$
}

\author{
Dong-Hyun Chae, Kyu-Ho Han, Kyung-Soo Lim, and Sun-Shin An \\ Department of Electronics Engineering, Korea University, \\ 1,5-ka, Anam-dong, Sungbuk-ku, Seoul, 136-701, Korea \\ \{hsunhwa, garget, angus, sunshin\}@dsys.korea.ac.kr
}

\begin{abstract}
We describe a problem of redundant flooding induced by multiple sensor nodes during route discovery in event-driven wireless sensor networks, and propose a novel extension to the on-demand ad hoc routing protocol, in order to reduce the number of signaling messages during the route discovery phase. That is, our extension reduces energy consumption during route discovery. The heuristically and temporarily selected Path Set-up Coordinator (PSC) plays the role of a route request broker that alleviates redundant route request flooding. The simulation results show that our extension not only helps to conserve energy, but also reduces the disruption caused by the broadcast storm.
\end{abstract}

\section{Introduction}

In this paper, we describe the broadcast storm problem [1] that is susceptible to arise during the route discovery phase as a result of redundant flooding from another viewpoint. This problem arises when several sensor nodes broadcast route request packets (RREQs) to the same destination (i.e., the sink node) in an event-driven sensor network in which the on-demand ad hoc routing protocol is utilized.

In an event-driven sensor network, several sensor nodes can detect an event simultaneously. The situation will inevitably arise in which multiple source nodes try to find route paths toward the same destination by RREQ flooding simultaneously in such a case. This redundant network-wide flooding causes considerable energy consumption and frequent MAC layer collision in a densely deployed sensor network.

Sensor nodes are energy constrained devices and therefore, it is crucial to minimize such redundant network-wide flooding. In order to overcome this problem, we propose a novel extension to the on-demand ad hoc routing protocol, in order to reduce the number of signaling messages during the route discovery phase.

\footnotetext{
* This work was partially supported by CUCN (National Center of Excellence in Ubiquitous Computing and Networking).
} 


\section{Our Proposal}

The main idea is very simple. If a node needs to obtain a route path to the sink node, it sends RREQs by the unicasting mechanism to the nearest node which has a route cache or which has a pending route discovery that was initiated by network-wide flooding.

Figure 1 shows a flow chart of our proposed algorithm. Figure 1-a) describes route discovery process and $b$ ) shows how to dispatch a received RREQ.

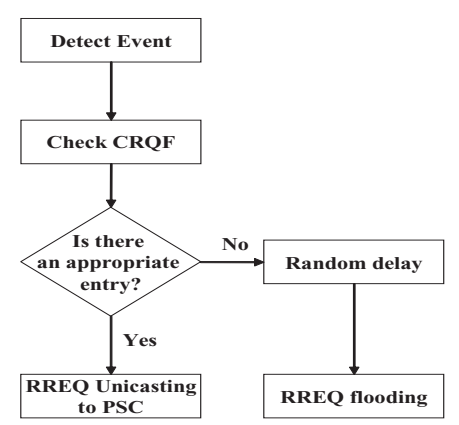

a) Route Discovery Process

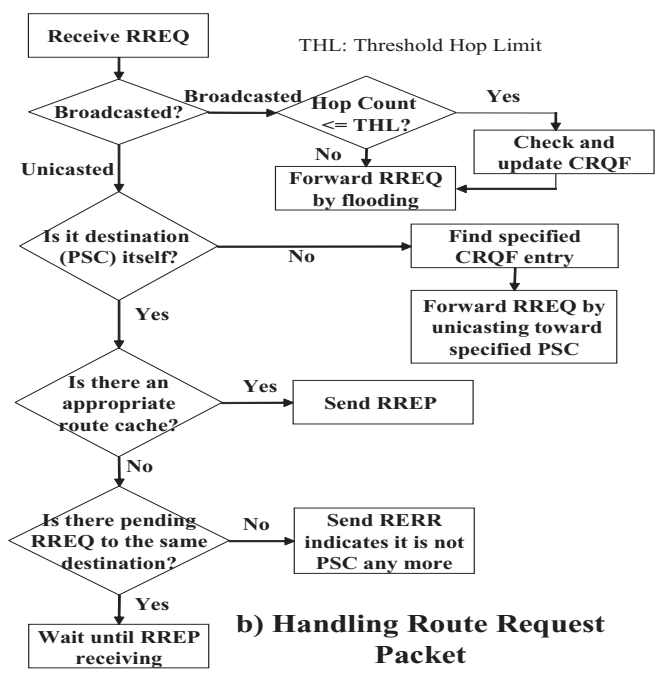

Fig. 1. Flow Chart of the Proposed Extension to the On-Demand Ad Hoc Routing Protocol

The Path Set-up Coordinator (PSC) is defined as the node that sends RREQs by network-wide flooding. All of the other neighbors and nodes which are located within the Threshold Hop Limit (THL) send RREQs by the unicast mechanism to the PSC.

The PSC is selected using a simple formula. It is simply the first node that tries to obtain a route path to the sink node in its locality. The PSC does not have permanent responsibility for replying to unicast RREQs. The PSC continues to perform this role, only as long as it has an active route cache. This heuristic approach is appropriate for the mechanism of Path Set-up Coordinator (PSC) selection. This approach eliminates the necessity to use an additional protocol and signaling message to select a PSC.

In order to be able to perform the unicast route set-up process, a node has to maintain a Cache of the RREQs that it receives by Flooding (CRQF). CRQF would need to contain the source node ID, timestamp, hop count, previous node ID or reverse source routes for source routing protocol, destination node ID (i.e. sink node ID) of the RREQ and current time. This entry can be managed by a soft-state policy and may be updated by another RREQ received by flooding. 


\section{$3 \quad$ Performance Evaluation}

We evaluated the proposed scheme using the ns-2 simulator [2], in order to evaluate its performance in terms of the energy conservation afforded by the reduction in the number of signaling messages.

We generated 10 topologies in an area of $200 \mathrm{~m}$ by $200 \mathrm{~m}$, in which 150 fixed nodes were randomly located. There was one randomly selected sink node in each topology.

We used the 802.11 MAC layer protocol and reduced the propagation range to $40 \mathrm{~m}$. The simulation time was limited to 100 seconds. We forced an event to move toward a randomly selected location. We selected AODV [3] as on-demand routing protocol.

We compared three different modes of the AODV as described below:

1. Default AODV (AODV): This is the default implementation of the AODV in ns-2. It is substantially implemented in Internet RFC 3561.

2. Modified Expanded Ring Search of AODV (MERS): Expanded Ring Search is implemented and utilized in Default AODV. We modified and adjusted this default expanded ring search implementation so that the strategy of the expanded ring search can be applied to the initial path setup phase without the necessity for the old path information.

3. AODV modified according to our suggestion (OURS): This is the extended implementation, incorporating our proposed mechanism. The proposed algorithm was implemented using the THL value that is indicated by $\mathrm{K}$ in table 1. As the value of $K$, that is THL, increases, the total number of the signaling messages decreases. However, if THL were larger, many nodes would not take the optimal route path. Therefore, the appropriate THL value should be selected on the basis of sufficient topology information.

The simulations were performed 10 times for each of the 10 different topologies and tables 1 shows the average values of each of the measured numerical factors, as described below:

1. Total Signaling Messages: This factor indicates the total number of signaling messages that are transmitted and received. (i.e., Total Tx + Total Rx)

2. Path Setup Success Ratio: This is the ratio of the total number of attempts to find paths successful or not to the total number of attempts.

3. Path Setup Latency: This means the path setup latency itself.

4. Path Setup Latency up to Success: This indicates the accumulated path setup latency from the initial path setup attempt until successful path setup is accomplished. If the initial path setup attempt fails, the node retries after a specified timeout.

Table 1 shows our simulation result. Using the proposed mechanism, reductions in the number of signaling messages of approximately $66.7 \%(\mathrm{~K}=1), 84.8 \%$ $(\mathrm{K}=2), 86.9 \%(\mathrm{~K}=3)$ and $88.6 \%(\mathrm{~K}=4)$ are obtained in comparison with default AODV and $45.5 \%(\mathrm{~K}=1), 75.2 \%(\mathrm{~K}=2), 78.5 \%(\mathrm{~K}=3)$ and $81.4 \%(\mathrm{~K}=4)$ packet reductions are observed in comparison to the MERS. 
Table 1. Simulation result

\begin{tabular}{|c|c|c|c|c|c|c|}
\hline & $\begin{array}{c}\text { Default } \\
\text { AODV }\end{array}$ & MERS & $\begin{array}{c}\text { OURS } \\
(\mathrm{K}=1)\end{array}$ & $\begin{array}{l}\text { OURS } \\
(\mathrm{K}=2)\end{array}$ & $\begin{array}{l}\text { OURS } \\
(\mathrm{K}=3)\end{array}$ & $\begin{array}{l}\text { OURS } \\
(\mathrm{K}=4)\end{array}$ \\
\hline \hline Total Signaling Messages & 31799.9 & 19428.4 & 10586.7 & 4826.8 & 4172.1 & 3618.6 \\
\hline Path Setup Success Ratio & 0.5471 & 0.4406 & 0.7116 & 0.8683 & 0.8063 & 0.7898 \\
\hline Path Setup Latency & 0.3307 & 0.1622 & 0.1473 & 0.1283 & 0.1293 & 0.2217 \\
\hline Path Setup Latency up to Success & 1.5062 & 0.8230 & 0.5310 & 0.3173 & 0.3244 & 0.5653 \\
\hline
\end{tabular}

The observed values of the path setup latency and the path setup latency up to success show that the proposed scheme causes the sensor nodes to take route paths with less setup latency. The path setup success ratio indicates that the proposed algorithm provides greater freedom from the broadcast storm problem, while simultaneously reducing the energy consumption.

\section{Conclusion}

The issue of energy conservation is critical in sensor networks, in which energy resources are limited. In this study, we propose a novel extension to the on-demand routing protocol for event-driven, randomly and densely deployed wireless sensor networks.

Our extension is designed to reduce redundant route request packets in eventdriven sensor networks. A Path Set-up Coordinator (PSC) is heuristically and temporally selected on demand, and it is only allowed to perform network-wide flooding in a local area. All the other nodes send route request packets to the PSC by unicasting.

This PSC selection and unicasting mechanism reduces the number of required signaling messages and this signaling reduction in turn lowers the energy consumption.

The simulation results demonstrate that the proposed extension can not only conserve energy, but can also reduce the disruption caused by the broadcast storm problem. The proposed protocol outperforms the other protocols in terms of both energy consumption and the successful route discovery rate, as well as lowering the path setup latency in the route discovery phase.

\section{References}

1. Y. Tseng, S. Ni, Y. Chen, and J. Sheu, "The Broadcast Storm Problem in Mobile Ad Hoc Network," Wireless Networks, Vol. 8, 153-167, 2002

2. NS-2 Network Simulator, http://www.isi.edu/nsnam/ns

3. C. Perkins, E. Belding-Royer, S. Das, "Ad hoc On-Demand Distance Vector (AODV) Routing," Internet RFC 3561, July 2003 